\section{PHYSICS AND RELATIVITY}

Relativity

Edited by. Moshe Carmeli, Stuart I. Fickler and Louis Witten. (Proceedings of the Relativity Conference in the Midwest, Cincinnati, Ohio, June 2-6, 1969.) Pp. xii +381 . (Plenum: New York and London, 1970.) $\$ 20 ; 180 s$.

IN 1963 R. K. Sachs said in a telling phrase, "General relativity is a legitimate, though minor, part of present fundamental physics . . . because at present it has few interactions with the remainder of physics". In this way he set the stage for a decade of development of the subject and provided a yardstick against which one can judge the steady stream of volumes of conference proceedings and collected papers which shows no sign of diminishing. The present volume reports on a national conference, but the diversity of activity in North America is sufficient to provide a view that reflects most of the recent developments in the subject.

Among others the quantization programme is reviewed by Komar and quantum gravity pursued by Klauder and Misner. A development which promises more relations with the rest of physics is foreshadowed in the Boltzmann theory paper of Anderson and in a note on the EinsteinLiouville equations by Berezdivin and Sachs. The experimental side is looked after by a report of Weber on his detection of radiation and a very valuable survey of the use of low temperature techniques by Everitt, Fairbank and Hamilton. Singularities are particularly well dealt with by Geroch, and the reader who wishes to whet his teeth may be directed towards Geroch's solution of twenty problems which he believes to be fairly difficult, and all unsolved, on pages 287-9. The fairly new topic of superspace is discussed firstly by Wheeler in a very characteristic paper called "Particles and Geometry", giving one of the usual wide-ranging views of possibilities in the subject, and in more detail by Fischer and DeWitt. Perhaps the most unusual paper is that entitled "Contribution to the History of Einstein's Geometry as a Branch of Physics" by Eugene Guth, which can be read with the greatest of interest by any physicist, including those who consider themselves experts in general relativity.

To what extent then has the subject become related with the rest of physics? The answer provided by this volume is, I think, that the process has definitely begun, but it is likely to be long and painful.

C. W. Kinmister worker has not had an adequate preparation in the necessary parts of the theory of radiation and of spectroscopy. This book attempts to bridge the gap between an intermediate level of college physics and the introductory stages of astrophysics. Four of the six chapters deal with relevant facets of the theory of matter and radiation, and two deal with astrophysics proper. Chapters one, three, five and six handle the theory of the line absorption coefficient, necessary parts of statistical mechanics, the semi-classical theory of line broadening, and the quantummechanical treatment of pressure broadening. Chapters two and four consider typical problems of spectral lines in stellar atmospheres and the classical methods of quantitative analysis of a stellar atmosphere. The book is completed with four appendices, a list of references, an extensive index of symbols used and their various definitions and a good general index.

The list of symbols used is particularly valuable because in many cases, when a particular facet of the physics of radiation is discussed, the notation of a standard textbook on the subject is maintained. This procedure leads to the same letter or symbol sometimes having two or more meanings as one proceeds through the book. Within any one section, however, the particular meaning adopted is there carefully preserved.

The author wishes his book to be a source of encouragement to the astronomer who wants to study stellar spectra without becoming too deeply involved in the non-equilibrium theory of transfer theory. This point of view is supported with statements such as "specifically we shall not concern ourselves with the details of the transfer of radiation within the spectral line" and "no one questions the use of Saha's theory of ionization in the interpretation of the spectral sequence".

I think that an introduction to astrophysics should attempt to sharpen the student's critical power by pointing out the weakness of argument underlying statements such as these and to encourage the student to think more deeply. If the student can be assumed to be prepared to accept, without preamble, derivation of the equation of transfer in general vector notation and to follow without undue struggle a discussion of theoretical line strengths (taken more or less directly from the text on Atomic Spectra by Condon and Shortley), he has the necessary analytical knowledge to profit by such an experience. He will then be prepared to advance towards the goal discerned by stellar spectroscopists so many years ago.
ANNE B. UNDERHILL

\title{
STELLAR SPECTROSCOPY
}

\section{The Theory of Stellar Spectra}

By Charles R. Cowley. (Topics in Astrophysics and Space Physics.) Pp. xi +260 . (Gordon and Breach: New York and London, May 1970.) 195s (\$23.40) boards; $70 s$ (\$8.50) paper.

For approximately 100 years, astronomers have been attempting to deduce detailed information about conditions in stellar atmospheres and the abundances of the chemical elements from the appearance of stellar spectra. The physical concepts used are, on the whole, relatively simple and straightforward. For their understanding they require chiefly a knowledge of the theory of radiation of optical wavelengths and how this radiation interacts with matter in a gaseous phase. Because of the way in which the subject developed, however, the terminology of astrophysics is complex and occasionally contradictory. The result is that he who would measure and analyse stellar spectra in order to understand stars and to find clues to the origin of the chemical elements and to the history of the galaxy often has a difficult time getting into his subject.

Many of the difficulties arise because the young research

\section{LIPID REVIEW}

\section{Topics in Lipid Chemistry}

Vol. 1. Edited by F. D. Gunstone. Pp. ix +451. (Logos, in association with Elek Books: London, July 1970.) $160 s$.

Thrs volume is the first of a series intended to provide a continuing review of the chemistry, physics and technology of lipids, without omitting important biological aspects. In addition, a form of information service is offered by appending a list of relevant books and reviews, but not original papers, published in the past eight years.

Not surprisingly, in an area where the introduction of physico-chemical procedures for separation and characterization of naturally occurring lipids has initiated a vast growth of publications, the chapters tend towards either techniques themselves or the application of techniques to a particular problem. Thus there are comprehensive and authoritative reviews of the use of gas liquid chromatography for the determination of structure of fatty acid esters, of mass spectrometry, and of hydrogenation catalysts. These subjects are clearly of value on account of their generality of application and interdisciplinary 